\title{
Association of patterns of care, prognostic factors, and use of radiotherapy-temozolomide therapy with survival in patients with newly diagnosed glioblastoma: a French national population- based study
}

\author{
Pascale Fabbro-Peray ${ }^{1,2}$ - Sonia Zouaoui ${ }^{3,4,5}$. Amélie Darlix ${ }^{5,6}$. Michel Fabbro ${ }^{5,6}$. Johan Pallud ${ }^{7}$. Valérie Rigau ${ }^{5,8}$. \\ Hélène Mathieu-Daude ${ }^{5,9}$. Faiza Bessaoud ${ }^{10}$. Fabienne Bauchet ${ }^{5}$. Adeline Riondel $^{1,2}$. Elodie Sorbets ${ }^{1,2}$. \\ Marie Charissoux 5,11 . Aymeric Amelot ${ }^{12} \cdot$ Emmanuel Mandonnet $^{12} \cdot$ Dominique Figarella-Branger $^{13}$. \\ Hugues Duffau ${ }^{3,4,5} \cdot$ Brigitte Tretarre $^{10} \cdot$ Luc Taillandier $^{14} \cdot$ Luc Bauchet $^{3,4,5}$ (D)
}

Received: 3 October 2018 / Accepted: 27 November 2018 / Published online: 6 December 2018

(c) The Author(s) 2018

\begin{abstract}
Background Glioblastoma is the most frequent primary malignant brain tumor. In daily practice and at whole country level, oncological care management for glioblastoma patients is not completely known.

Objectives To describe oncological patterns of care, prognostic factors, and survival for all patients in France with newlydiagnosed and histologically confirmed glioblastoma, and evaluate the impact of extended temozolomide use at the population level.

Methods Nationwide population-based cohort study including all patients with newly-diagnosed and histologically confirmed glioblastoma in France in 2008 and followed until 2015.

Results Data from 2053 glioblastoma patients were analyzed (male/female ratio 1.5, median age 64 years). Median overall survival (OS) was 11.2 [95\% confidence interval (CI) 10.7-11.9] months. The first-line therapy and corresponding median survival (MS, in months) were: $13 \%$ did not receive any oncological treatment (biopsy only) (MS =1.8, 95\% CI 1.6-2.1), $27 \%$ received treatment without the combination of radiotherapy (RT)-temozolomide (MS $=5.9,95 \%$ CI 5.5-6.6), 60\% received treatment including the initiation of the concomitant phase of RT-temozolomide (MS $=16.4,95 \%$ CI 15.2-17.4) whom $44 \%$ of patients initiated the temozolomide adjuvant phase (MS $=18.9,95 \%$ CI 18.0-19.8). Only $22 \%$ patients received 6 cycles or more of adjuvant temozolomide ( $\mathrm{MS}=25.5,95 \% \mathrm{CI} 24.0-28.3)$. The multivariate analysis showed that the risk of mortality was significantly higher for the non-progressive patients who stopped at 6 cycles (standard protocol) than those who continued the treatment, hazard ratio $=1.5(95 \%$ CI 1.2-1.9).

Conclusion In non-progressive patients, prolonging the adjuvant temozolomide beyond 6 cycles may improve OS.
\end{abstract}

Keywords Clinical epidemiology $\cdot$ Glioblastoma $\cdot$ Neuro-oncology $\cdot$ Neurosurgery $\cdot$ Population-based study

Temozolomide

With the participation of: Club de Neuro-Oncologie and Société Française de Neurochirurgie, Société Française de Neuropathologie, and Association des Neuro-Oncologues d'Expression Française.

Pascale Fabbro-Peray and Sonia Zouaoui have contributed equally to this article.

Electronic supplementary material The online version of this article (https://doi.org/10.1007/s11060-018-03065-z) contains supplementary material, which is available to authorized users.

Extended author information available on the last page of the article

\section{Introduction}

Glioblastoma multiforme (GBM) is the most frequent malignant primitive brain tumor and the most deadly glioma subtype [1-3]. In daily practice, oncological care management for GBM patients is not completely standardized and depends on many factors [age, Karnofsky performance status (KPS), comorbidity, tumor (location, shape, and volume), etc.]. Few studies have directly led to improvements in medical care [4-10]. Since 2005, one pivotal clinical trial 
defined the radiotherapy and temozolomide combination (RT-temozolomide) as the standard of care in patients with newly-diagnosed GBM, for patients 18-70 years old with a WHO performance status of $\leq 2$ [6]. Carmustine wafer $(\mathrm{CW})$ is an option when maximal safe resection (RS) is performed and high grade glioma is histologically proven [11-14]. More recently, bevacizumab in combination with RT-temozolomide in first-line treatment showed a significant prolonged progression-free survival but failed to demonstrate a significant overall survival (OS) benefit $[15,16]$. Moreover, "standards of care" do not exist for progression/ recurrent GBM patients (e.g., bevacizumab was approved for treatment of recurrent GBM in USA, but not in Europe). One paper has shown the dissemination of RT-temozolomide combination as the treatment standard across countries, and a modest survival gain at the population level [17].

This work is requested by the French government and describes the oncological patterns of care, prognostic factors, and survival for all patients with newly-diagnosed and histologically confirmed GBM, for a country with more than 60 million inhabitants. This first paper aims to answer several questions concerning the first-line therapy at the national level: What surgery was performed (biopsy only, partial RS, total RS with or without CW implantation)? What extent of combined RT-temozolomide treatment did patients receive (concomitant phase only, initiation of adjuvant phase, 6 cycles of temozolomide in maintenance or more)? What was the survival for the different patient groups and did it differ between the group of patients with exactly 6 versus $>6$ cycles of the adjuvant temozolomide treatment?

\section{Methods}

The French Brain Tumor DataBase (FBTDB) identified and recorded all patients with newly-diagnosed and histologically confirmed primary brain tumors (e.g., GBM) since 2006 in France, and prospectively collected initial data. FBTDB is one of the largest clinical databases for brain tumors in Europe [18-26].

\section{Study population}

This study includes all patients with newly-diagnosed and histologically confirmed GBM in 2008 (1 January-31 December). Histological diagnosis of GBM according to WHO 2007 classification [27] including glioblastoma, giant cell glioblastoma, and gliosarcoma, corresponding to ICD-O codes $9440 / 3,9441 / 3$, and 9442/3 respectively, were included. Exclusion criteria were previous surgery for GBM, spinal cord GBM, and patients from abroad or French overseas departments. A data card was used to retrospectively collect data on the oncological management [surgery, RT, and chemotherapy (CT)] and the followup care of these patients for the period 2008/01/01 to 2015/02/03. According to French neurosurgical guidelines, extent of RS should be evaluated with postoperative MRI. In our study, this information was reported from patient file. Central review was not performed.

The study was approved by the French legislation (CCTIRS n ${ }^{\circ} 10.548$; CNIL n $^{\circ}$ 911013).

\section{Treatment given}

The start and end dates for RT and total dose were requested. For CT, the start and end dates and name and modality of administration were also requested. For the study of the RT-temozolomide treatment, the concomitant and the adjuvant phases were analyzed separately, and the number of cycles of adjuvant temozolomide was noted. Biopsy was considered a surgical procedure but not a treatment procedure.

\section{Statistical analysis}

Statistical analysis was performed using SAS Enterprise Guide software, version 6.1. The analysis included a descriptive part of the original data and the oncological treatments received by the patients. Survival was estimated by the Kaplan-Meier method and defined as the time from first surgery (corresponding to the histological diagnosis) to death or censored at the date of last follow-up. The log-rank test was used to compare survival curves among different strata. The assumption of proportional hazards was verified before survival curve comparisons. Univariate Cox regression model was used to estimate hazard ratios (HRs) of strata versus reference level and their 95\% Wald confidence interval (CI). For continuous variables, log linearity was tested before HR estimations. Multivariate Cox regression model was used to determine independent prognostic factors of OS. Potential prognostic factors and interaction terms were introduced into the model according to the univariate analysis ( $\mathrm{p}$-entry $=0.20$ ), and a backward selection strategy was applied. We regarded $\mathrm{p}$ values $<0.05$ as statistically significant. The Bonferroni correction was used for the multiple comparison tests. In order to assess duration of adjuvant phase of the RT-temozolomide combination as a prognostic factor, after 6 cycles of temozolomide without progression, patients with the treatment termination information as "end of treatment as defined by the Stupp protocol" and all the patients $>6$ cycles, were selected and analyzed according to the same statistical strategy. 


\section{Results}

Of the 2167 cases identified by FBTDB, 114 were excluded (89 recurrences, 6 other histologic diagnoses, 4 duplicates and 15 patients from abroad). Therefore, this study included 2053 newly-diagnosed and histologically confirmed GBM patients. Of the 54 participating neurosurgical departments, 44 were public centers (36 university hospitals, 2 army hospitals, 4 general hospitals, and 2 nonprofit institutions) and 10 were private institutions; the majority of patients $(93.8 \%)$ were treated in public centers.

\section{Population characteristics}

Median age at diagnosis was 64 years and more than a quarter of patients were $>72$ years. Median preoperative KPS was $80 \%$ (range 20-100\%). Preoperative KPS was available for only 916 patients (45\%). Histological diagnosis was obtained during the initial surgery (RS 59\%, biopsy 41\%) (Table 1).

\section{Overall survival and main spontaneous prognostic factors}

Median OS was 11.2 (95\% CI 10.7-11.9) months, [13.6 (95\% CI 12.7-14.7) and 6.8 (95\% CI 5.8-7.5) months for patients $\leq 70$ and $>70$ years old, respectively]. Survival rates at $1 / 1.5 / 2 /$ and 5 years were: $47.1 \%$ (95\% CI $44.8-49.3 \%) / 31.4 \%$ (95\% CI 29.3-33.5\%)/20.1\% (95\% CI $18.3-22.0 \%$ )/and $4.5 \%$ (95\% CI 3.6-5.6\%) (Supplementary Fig. S1). The main prognostic factors were age, tumor location, and KPS. Notably: the median survival (MS) of the group "KPS reported" versus "KPS unreported" was not significantly different (MS $=11.7$ vs. 10.9 months, $\mathrm{p}=0.50)$ (Supplementary Fig. S2).

\section{Oncological management in first-line therapy}

Complete treatment information (including lack of treatment) was available for 1856 patients (Table 2). Nearly $60 \%$ of all patients initiated the concomitant phase of the combined RT-temozolomide treatment (group 1, $\mathrm{n}=1111)$. In this group, RS was performed in 805 patients $(72.5 \%)$, and 107 patients $(9.6 \%)$ received $\mathrm{CW}$ implantation. Nearly $45 \%$ of all patients $(n=821)$ initiated the adjuvant phase, and only $21.6 \%$ of all patients $(n=401)$ received 6 cycles or more of temozolomide during the adjuvant phase. Among all patients, approximately $40 \%$ did not receive the combined RT-temozolomide treatment
Table 1 Clinical characteristics of the 2053 patients at baseline

\begin{tabular}{|c|c|c|}
\hline Characteristic (no. reported) & $\mathrm{N}$ & $\%$ \\
\hline \multicolumn{3}{|l|}{ Sex (2053) } \\
\hline Male & 1232 & 60.0 \\
\hline Female & 821 & 40.0 \\
\hline \multicolumn{3}{|c|}{ Age per quartile, in years (2053) } \\
\hline$\leq 56$ & 515 & 25.1 \\
\hline $57-63$ & 460 & 22.4 \\
\hline $64-72$ & 547 & 26.6 \\
\hline$>72$ & 531 & 25.9 \\
\hline \multicolumn{3}{|l|}{ Signs and symptoms (2046) } \\
\hline Epilepsy & 450 & 22.0 \\
\hline Headache & 655 & 32.0 \\
\hline High intracranial pressure & 285 & 13.9 \\
\hline Mental status disorders & 930 & 45.4 \\
\hline Sensory-motor deficit & 924 & 45.2 \\
\hline Other & 352 & 17.2 \\
\hline \multicolumn{3}{|c|}{$\begin{array}{l}\text { Time between first sign and histological diagnosis, in months } \\
\text { (1667) }\end{array}$} \\
\hline$<1$ & 784 & 47.0 \\
\hline $1-2$ & 346 & 20.8 \\
\hline $2-3$ & 270 & 16.2 \\
\hline $3-4$ & 119 & 7.1 \\
\hline$\geq 4$ & 148 & 8.9 \\
\hline \multicolumn{3}{|l|}{ Preoperative KPS (916) } \\
\hline $90-100 \%$ & 456 & 49.8 \\
\hline $70-80 \%$ & 338 & 36.9 \\
\hline$\leq 60 \%$ & 122 & 13.3 \\
\hline \multicolumn{3}{|l|}{ Location of the tumor (1869) } \\
\hline Right & 880 & 47.1 \\
\hline Left & 855 & 45.7 \\
\hline Median and/or bilateral & 134 & 7.2 \\
\hline \multicolumn{3}{|c|}{ Modality of the histological diagnosis (first surgery) (2053) } \\
\hline “Total” RS & 476 & 23.2 \\
\hline Partial RS & 422 & 20.5 \\
\hline NOS RS & 309 & 15.1 \\
\hline Biopsy & 846 & 41.2 \\
\hline \multicolumn{3}{|l|}{ Histological diagnosis (2053) } \\
\hline Glioblastoma & 1988 & 96.8 \\
\hline Giant cell glioblastoma & 36 & 1.8 \\
\hline Gliosarcoma & 29 & 1.4 \\
\hline
\end{tabular}

KPS Karnofsky performance status, NOS not otherwise specified, $R S$ resection

(group 2; $\mathrm{n}=745$ ) including 372 patients $(20 \%)$ who did not receive any oncological treatment after surgery.

\section{Survival according to the first-line therapy}

Overall, the two main therapeutic prognostic factors were extent of RS and combined RT-temozolomide treatment 
Table 2 First-line therapy $(\mathrm{N}=1856)$

\begin{tabular}{|c|c|c|}
\hline First-line therapy ${ }^{\mathrm{a}, \mathrm{b}}$ & $\mathrm{N}$ & $\%$ \\
\hline $\begin{array}{l}\text { Group 1: first-line including combined radiotherapy and temozolomide (RT-temozolo- } \\
\text { mide) treatment }\end{array}$ & 1111 & 59.9 \\
\hline Surgery + RT-concomitant temozolomide initiated & 1111 & \\
\hline RS + RT-concomitant temozolomide initiated & 805 & \\
\hline RS without $\mathrm{CW}$ & 698 & \\
\hline RS with CW & 107 & \\
\hline $\mathrm{B}+\mathrm{RT}$-concomitant temozolomide initiated & 306 & \\
\hline Surgery + RT-concomitant temozolomide + adjuvant temozolomide initiated & 821 & 44.2 \\
\hline RS + RT-concomitant temozolomide + adjuvant temozolomide initiated & 638 & \\
\hline RS without CW & 561 & \\
\hline RS with CW & 77 & \\
\hline B + RT-concomitant temozolomide + adjuvant temozolomide initiated & 183 & \\
\hline Surgery + RT - concomitant temozolomide + adjuvant temozolomide $<6$ cycles & 420 & 22.6 \\
\hline $\mathrm{RS}+\mathrm{RT}-$ concomitant temozolomide + adjuvant temozolomide $<6$ cycles & 308 & \\
\hline $\mathrm{RS}$ without $\mathrm{CW}$ & 275 & \\
\hline RS with CW & 33 & \\
\hline $\mathrm{B}+\mathrm{RT}-$ concomitant temozolomide + adjuvant temozolomide $<6$ cycles & 112 & \\
\hline Surgery + RT-concomitant temozolomide + adjuvant temozolomide $=6$ cycles $^{\mathrm{c}}$ & 220 & 11.9 \\
\hline RS + RT - concomitant temozolomide + adjuvant temozolomide $=6$ cycles & 190 & \\
\hline $\mathrm{RS}$ without $\mathrm{CW}$ & 162 & \\
\hline RS with CW & 28 & \\
\hline $\mathrm{B}+\mathrm{RT}-$ concomitant temozolomide + adjuvant temozolomide $=6$ cycles & 30 & \\
\hline Surgery + RT-concomitant temozolomide + adjuvant temozolomide $>6$ cycles & 181 & 9.8 \\
\hline $\mathrm{RS}+\mathrm{RT}$-concomitant temozolomide + adjuvant temozolomide $>6$ cycles & 140 & \\
\hline $\mathrm{RS}$ without $\mathrm{CW}$ & 124 & \\
\hline RS with CW & 16 & \\
\hline $\mathrm{B}+\mathrm{RT}-$ concomitant temozolomide + adjuvant temozolomide $>6$ cycles & 41 & \\
\hline Group 2: first-line excluding combined RT-temozolomide treatment & 745 & 40.1 \\
\hline B alone (no treatment) & 241 & 13.0 \\
\hline RS alone (no other treatment) & 131 & 7.1 \\
\hline $\mathrm{B}+\mathrm{CT}$ & 156 & 8.4 \\
\hline Temozolomide 5/28 & 118 & \\
\hline Bevacizumab & 15 & \\
\hline Other CT & 23 & \\
\hline $\mathrm{B}+\mathrm{RT}$ & 53 & 2.9 \\
\hline $\mathrm{RS}+\mathrm{CT}$ & 66 & 3.6 \\
\hline Temozolomide 5/28 & 38 & \\
\hline Bevacizumab & 6 & \\
\hline Other CT & 22 & \\
\hline $\mathrm{RS}+\mathrm{RT}$ & 70 & 3.8 \\
\hline Other & 28 & \\
\hline
\end{tabular}

$B$ biopsy, $C T$ chemotherapy, CTconco concomitant chemotherapy, $C W$ carmustine wafer, $R S$ resection, $R T$ radiotherapy

${ }^{a}$ Median duration and median dose for RT were 44 days and 60 Gy respectively

${ }^{\mathrm{b}}$ Median times from surgery to the next treatment with RT-temozolomide, CT alone, or RT alone were 39, 20, 40 days respectively

${ }^{c}$ Of the 220 patients who received exactly 6 cycles of adjuvant temozolomide, the treatment termination information for "end of treatment as defined by the Stupp protocol" was specified in 166 cases 
(Fig. 1a, b). Analysis of survival according to the first surgery (total RS, partial RS, not otherwise specified RS and biopsy) showed significant differences in MS, as follows: 18.1 (95\% CI 17.2-19.2), 12.3 (95\% CI 11.1-13.1), 11.9 (95\% CI 10.1-14.6), and 5.8 (95\% CI 5.4-6.5) months respectively, $\mathrm{p}<0.001$, log-rank test. Analysis of survival of group 1 versus 2 (according to first-line therapy including or excluding the combined RT-temozolomide treatment) showed significant differences in MS, as follows: MS for group $1=16.4$ (95\% CI 15.2-17.4) months and MS for group $2=4.1$ (95\% CI 3.7-4.6) months, $\mathrm{p}<0.001$, log-rank test. But, there were significant differences in patient characteristics between the groups. Patients in group 1 were significantly younger, with less median and bilateral lesion, with better KPS, with more RS versus biopsy, and with more total RS versus other RS than group 2 (Supplementary Table 1). After adjusting for age in quartiles and type of first surgery, mortality was higher for treatment without RT-temozolomide versus treatment with $\mathrm{RT}$-temozolomide, $\mathrm{HR}=2.8$ (95\% CI 2.5-3.1) (Supplementary Table 2). When KPS was introduced into the model as reported value $(n=861$ patients) and/or as missing value ( $\mathrm{n}=1856$ patients), results were very similar (Supplementary Table 3).

Survival in group 1 according to the surgery $(\mathrm{RS}, \mathrm{n}=805$ vs. biopsy, $n=306)$ showed a significant difference: MS for group 1 with $\mathrm{RS}=17.8$ (95\% CI 17.0-18.8) and MS for group 1 with biopsy $=12.2$ (95\% CI 11.2-13.1) months, $\mathrm{p}<0.001$, log-rank test (Fig. 1c).

Survival in the RS group ( $\mathrm{n}=805)$, according to the local $\mathrm{CT}$, when analysis is limited to the patients with the information of total or subtotal $\mathrm{RS}(\mathrm{n}=482,79$ patients received CW and 403 did not receive CW), MS were 22.6 (95\% CI 17.1-28.4) and 18.5 (95\% CI 17.4-19.6) months, respectively, $\mathrm{p}=0.19$, log-rank test (Fig. 1d).

OS for the 821 patients who initiated the adjuvant phase was 18.9 (95\% CI 18.0-19.8) months. Among them, 401 patients received 6 cycles or more of adjuvant temozolomide (MS $=25.5,95 \%$ CI 24.0-28.3 months). According to the number of received cycles of adjuvant temozolomide $(<6$ cycles: $n=420,=6$ cycles: $n=220,>6$ cycles: $n=181$ ), MS showed significant difference and were: $12.8(95 \% \mathrm{CI}$ 12.2-13.8), 23.8 (95\% CI 21.6-26.6), and 29.8 (95\% CI 25.1-32.5) months, respectively, $\mathrm{p}<0.001$, log-rank test (Fig. 1e). Pairwise comparisons were statistically significant, $\mathrm{p}<0.001$ for all comparisons. Survival in the subgroups " $<6,=6,>6$ cycles", according to the surgery (RS vs. biopsy) is shown in Supplementary Fig. S3.

Of the 220 patients who received strictly 6 cycles of adjuvant temozolomide, the treatment termination information for "end of treatment as defined by the standard treatment protocol" [6] (and not for progression or other reason) was specified in 166 cases. Analysis of survival based on discontinuation of the adjuvant temozolomide after the 6 th cycle based on the definition of the protocol versus the continuation until progression showed significant differences in MS, as follows: 27.2 (95\% CI 23.8-28.8) and 29.8 (95\% CI 25.1-32.5) months, respectively, $\mathrm{p}=0.008$, log-rank test (Fig. 1f). These two subgroups were not significantly different for age or KPS, whereas the subgroup "continuation" (with the higher MS) received significantly less total RS (Table 3). After adjustment for surgery (total RS vs. other), the risk of mortality was significantly higher for patients who stopped at 6 cycles than those who continued the treatment, HR $=1.5$ (95\% CI 1.2-1.9) (Table 4).

MS in group $2(n=745)$ was $4.1(95 \%$ CI 3.7-4.6) (Fig. 1b) and 5.9 (95\% CI 5.5-6.6) months when patients with biopsy only were excluded. Main subgroup MS (months) were: 1.8 (95\% CI 1.6-2.1) for biopsy only, 5.3 (95\% CI 4.8-6.5) for biopsy + CT, 7.2 (95\% CI 5.2-8.7) for biopsy + RT, 6.8 (95\% CI 5.9-9.1) for RS + CT, and 10.8 (95\% CI 9.4-12.9) for RS + RT.

\section{Discussion}

This study, including all patients with newly-diagnosed and histologically confirmed GBM, detailed the proportions of patients who: (1) initiated the standard treatment, (2) initiated the adjuvant phase, (3) received strictly 6 or $>6$ cycles of adjuvant TMZ before progression. MS were estimated for each group, and we showed that prolonging the adjuvant temozolomide beyond 6 cycles positively impacted survival in non-progressive patients.

In our study, the crude incidence rate was 3.3/100,000 person-years and is in good accordance with the literature [1]. OS was 11.2 months, similar to the values obtained in population-based studies in the post-temozolomide era [28-30]. Survival rates were similar to US results [2], and intermediate between those of the RT-only arm and those of the RT-temozolomide arm in the EORTC-NCIC clinical trial [31]. Woehrer et al. reviewed GBM survival in different population studies and showed a significant but modest survival gain over time [17]. We can explain this modest survival gain: (i) in "real world", only $60 \%$ of all patients initiate the standard treatment, (ii) less than a quarter of all patients received 6 cycles or more of adjuvant temozolomide, and (iii) elderly patients have been treated more often in recent years.

Some previous papers have summarized the first-line treatment in population studies. For example, after surgery in the works of Graus et al. [32], Brandes et al. [33], and in our series, $57 \% / 62.5 \% / 59.9 \%$ of all patients received the combined RT-temozolomide treatment, 21\%/15.7\%/20.1\% received other regimens, and 22\%/21.7\%/20\% were not treated, respectively. But, to our knowledge, only one recent paper described the detail and outcome of the different 

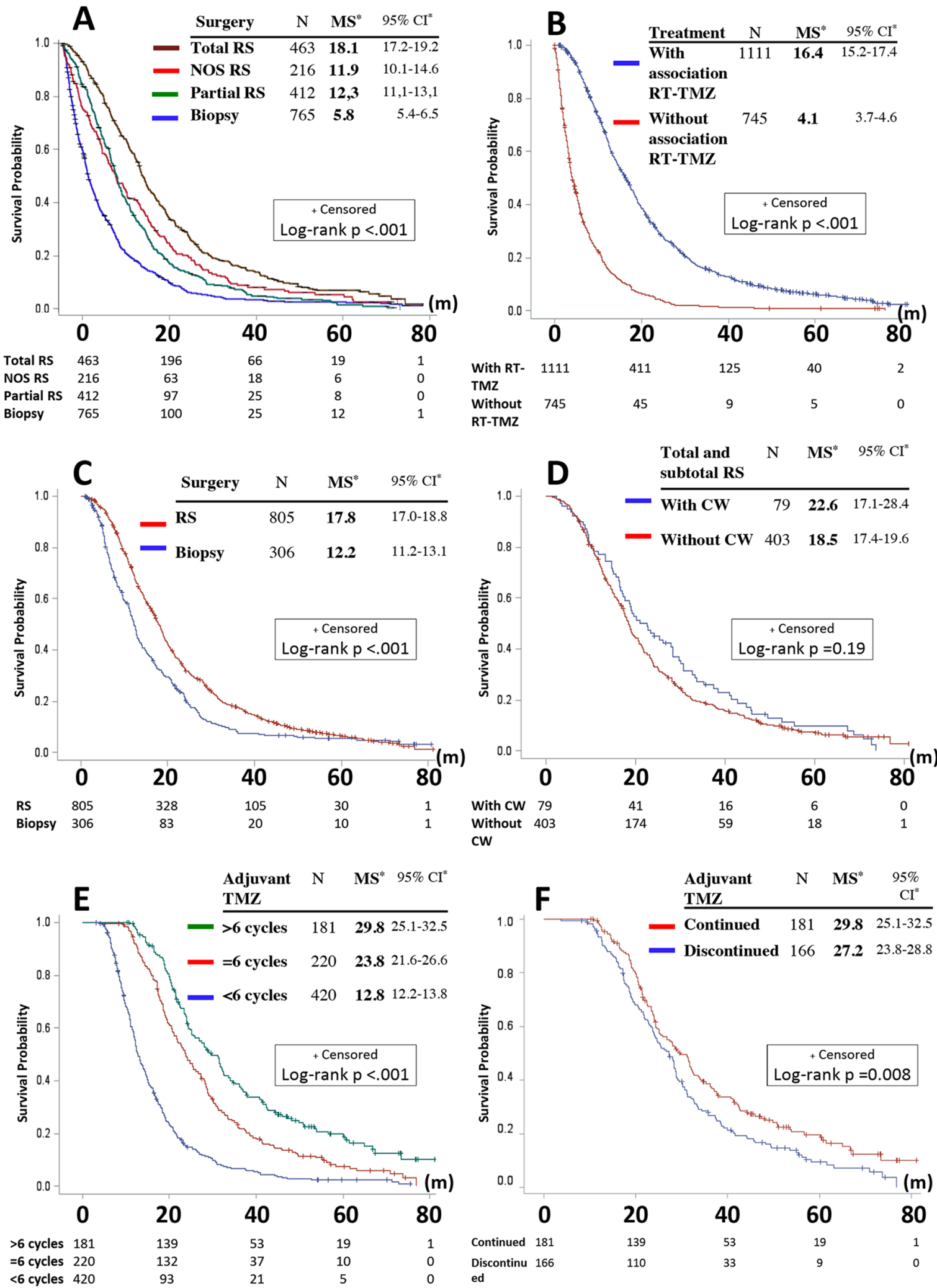
४Fig. 1 Survival and treatment patterns. Kaplan-Meier estimates of survival by: a first surgery (total RS, partial RS, NOS RS, and biopsy), $\mathbf{b}$ treatment including or excluding the combination of radiotherapy and temozolomide (RT-TMZ) in first-line treatment, c surgery (RS vs. biopsy) in the group of patients who initiated (at least) the RT-TMZ, d treatment with versus without local chemotherapy (carmustine wafer, $\mathrm{CW}$ ) in the group of patients with total or subtotal RS and who initiated (at least) the RT-TMZ, e number of cycles of temozolomide (TMZ) $(<6,=6,>6 \mathrm{c})$ in the group of patients who received adjuvant TMZ, $\mathbf{f}$ continuation versus discontinuation of the adjuvant TMZ after 6 cycles as defined by the Stupp protocol (in the group of patients free of progression). *Median survival (MS) and confidence interval (CI) are expressed in months (m). $c$ Cycle, NOS not otherwise specified, $R S$ resection

phases of the standard protocol in a large population-based study, and according to the surgery [30]. The results from the Danish population-based study and our results (main spontaneous prognostic factors, OS, survival according to the surgery and to postoperative treatment, etc.) were very close. This highlights the interest of nationwide population studies.

\section{Survival according to the duration of maintenance therapy of temozolomide}

The question of the optimal duration of maintenance therapy of temozolomide remains controversial. Some institutions stop the adjuvant temozolomide after 6 cycles in accordance with the protocol, whilst others prolong treatment up to 12 or even more cycles in non-progressive patients. In daily practice, the prescribed number of cycles for patients without tumor progression after 6 months varies greatly. Many previous and ongoing trials of CT for malignant gliomas prescribe maintenance temozolomide for up to 12 months [34-36]. Early publications on this subject claimed prolonged survival of patients receiving temozolomide treatment extended beyond 6 cycles [37-40]. In contrast, three recent interesting works concluded that continuing temozolomide beyond 6 cycles does not show longer OS [35, 41, 42]. But none of them was a specific clinical trial or a real population-based study. Blumenthal et al. [35] performed an interesting pooled analysis of individual patient data from four randomized trials for newly diagnosed GBM. A total of 2214 GBM patients were included in the four trials. All patients who were progression free 28 days after cycle 6 were included. The decision to continue temozolomide was per local practice and standards, and at the discretion of the treating physician. Of these, 624 qualified for analysis: 291 continued maintenance temozolomide until progression or up to 12 cycles, while 333 discontinued temozolomide after 6 cycles (these nearly equivalent numbers illustrate the difficulty of the question). First, as noted by the authors, this study is an unplanned retrospective meta-analysis of patients included in clinical trials spanning a decade. Secondly, these patients were selected and were not representative of the population in the real world (age, percentage of complete RS, comorbidity, etc.). Thirdly, whilst the percentage of approximately $30 \%$ of patients remained progression free at the end of the standard 6 cycles of temozolomide maintenance therapy is in accordance with data from clinical trials, it is high compared with data from population-based or real world studies. This could reflect the frequent selectivity of patients in inclusion in clinical trials. Fourthly, the pattern of patients free of progression after the 6th adjuvant temozolomide, includes in these randomized studies was different than ours, particularly in proportion of total RS (53.7 vs. $42.7 \%$ ). This better prognostic factor is less frequent in real life population patients and participates to increase the heterogeneity [30]. We can speculate that prolonging temozolomide beyond 6 cycles could offer benefit in OS to a subset of patients that could not have been operated in a maximal approach (for different reason: age, comorbidity, tumor topography, etc.). But unfortunately, our work does not allow us to identify which subgroup of patients benefit most from the continuation of treatment.

\section{Note concerning the French oncological management for glioblastoma}

There is not typical peculiarity. However, firstly, we can notice that $6.2 \%$ of the patients only, were operated in private neurosurgical centers while French hospitals (in general) include $33 \%$ of private centers (Direction générale de l'offre de soins, DGOS, https://solidarites-sante.gouv. fr/IMG/pdf/dgos_cc_2018_02_16_a_web_pages_hd.pdf). This difference can be explained by the fact that there is a very strict regulation for the brain tumors management in France. Secondly, here, the percentage of RS (59\%) is lower than in many studies. But most data come from clinical trials or studies from specific centers, while accurate neurosurgical data from population-based studies are limited. Thirdly, the management (median dose $60 \mathrm{~Gy}$ ) and the percentage of patients who received RT as first-line treatment (68\%) were similar as in many countries. Fourthly, concerning the duration of maintenance therapy of temozolomide before recurrence, no recommendation does exist in France. Some centers treated with 6 cycles strictly while other treated during 1 year, or even longer.

\section{Limitations}

Our study did not include any biological data [i.e., $\mathrm{O}_{6}$-methylguanine DNA methyltransferase (MGMT) promoter methylation status, or isocitrate dehydrogenase (IDH) mutation status], nor the presence of residual tumor after the 6th cycle (e.g., our study did not provide information on the reasons why the temozolomide was continued, 
Table 3 Comparison of main characteristics of patients receiving 6 cycles as defined by the protocol [sub-group 1, of the 220 patients who received strictly 6 cycles of adjuvant temozolomide, the treatment termination information for "end of treatment as defined by the protocol" (and not for progression or other reason) was specified in 166 cases] versus more than 6 cycles (sub-group 2 ) of adjuvant temozolomide

\begin{tabular}{|c|c|c|c|}
\hline Characteristic (no. reported) & $\begin{array}{l}\text { Sub-group } 1 \text { "discontinued" } \\
(\mathrm{N}=166) \mathrm{n}(\%)\end{array}$ & $\begin{array}{l}\text { Sub-group } 2 \text { "continued" } \\
(\mathrm{N}=181) \mathrm{n}(\%)\end{array}$ & $\mathrm{p}$ \\
\hline Age per quartile, in years (347) & & & 0.61 \\
\hline$\leq 56$ & $65(39.16)$ & $83(45.86)$ & \\
\hline $57-63$ & $45(27.11)$ & 47 (25.97) & \\
\hline $64-72$ & 39 (23.49) & $36(19.89)$ & \\
\hline$>72$ & $17(10.24)$ & $15(8.29)$ & \\
\hline Preoperative KPS (163) & (Missing data: 102) & (Missing data: 82) & 0.92 \\
\hline $90-100 \%$ & $42(65.63)$ & $63(63.64)$ & \\
\hline $70-80 \%$ & 19 (29.69) & $30(30.30)$ & \\
\hline$\leq 60 \%$ & $3(4.69)$ & $6(6.06)$ & \\
\hline First surgery (347) & & & $<0.001$ \\
\hline “Total” RS & $91(54.82)$ & $57(31.49)$ & \\
\hline Partial RS & $38(22.89)$ & $57(31.49)$ & \\
\hline NOS RS & $18(10.84)$ & $26(14.36)$ & \\
\hline Biopsy & $19(11.45)$ & $41(22.65)$ & \\
\hline First surgery (347) & & & $<0.001$ \\
\hline "Total” RS & $91(54.82)$ & $57(31.49)$ & \\
\hline Other surgery & $75(45.18)$ & $124(68.51)$ & \\
\hline
\end{tabular}

KPS Karnofsky performance status, NOS not otherwise specified, $R S$ resection

Table 4 Relative risk of mortality: uni- and multi-variate analysis for patients who discontinued temozolomide as defined by the protocol and for patients who continued temozolomide until progression

\begin{tabular}{|c|c|c|c|c|c|c|}
\hline \multirow[t]{2}{*}{ Variables } & \multirow[t]{2}{*}{$\mathrm{N}$} & \multirow{2}{*}{$\begin{array}{l}\mathrm{Nb}(\%) \text { of } \\
\text { deceased patients }\end{array}$} & \multicolumn{2}{|l|}{ Univariate analysis } & \multicolumn{2}{|l|}{ Multivariate analysis } \\
\hline & & & Hazard ratio $(95 \% \mathrm{CI})$ & $\mathrm{p}$ values & Hazard ratio $(95 \% \mathrm{CI})$ & $\mathrm{p}$ values \\
\hline Treatment & & & & 0.009 & & 0.001 \\
\hline $\begin{array}{l}\text { "Continued" (>6 } \\
\text { cycles of adjuvant } \\
\text { temozolomide) }\end{array}$ & 181 & $139(76.8)$ & 1 & & 1 & \\
\hline $\begin{array}{l}\text { "Discontinued" (=6 } \\
\text { cycles of adjuvant } \\
\text { temozolomide) }\end{array}$ & 166 & $147(88.6)$ & $1.364(1.082-1.721)$ & & $1.493(1.172-1.903)$ & \\
\hline Age (years) & & & & 0.07 & & $\begin{array}{l}\text { Excluded } \\
\text { from model } \\
(\mathrm{NS})\end{array}$ \\
\hline$\leq 56$ & 148 & $120(81.1)$ & 1 & & & \\
\hline $57-63$ & 92 & $72(78.3)$ & $1.051(0.785-1.408)$ & & & \\
\hline $64-72$ & 75 & $64(85.3)$ & $1.391(1.026-1.884)$ & & & \\
\hline$>72$ & 32 & $30(93.8)$ & $1.485(0.994-2.219)$ & & & \\
\hline Surgery & & & & 0.10 & & 0.01 \\
\hline Total resection & 148 & $119(80.4)$ & 1 & & 1 & \\
\hline Other surgery & 199 & $167(83.9)$ & $1.218(0.963-1.542)$ & & $1.368(1.070-1.749)$ & \\
\hline
\end{tabular}

such as the persistence (or not) of enhanced lesions on imaging, or the knowledge of the MGMT status). Therefore, the main limitation of our study is that we cannot formally eliminate the existence of a bias in the result of the survival analysis according to the duration of maintenance therapy of temozolomide.
However, for the comparison of the survival between the two groups (patients who received strictly 6 cycles of adjuvant temozolomide vs. patients who received $>6$ cycles), we included only patients with the information of "end of treatment as defined by the standard treatment protocol" and not for progression or other reason, in the 
group with strictly 6 cycles. Moreover, these two groups were not significantly different for age or KPS, whereas the group "continuation" (with the higher MS) received significantly less total RS (Table 3). The multivariate model showed that the risk of mortality was significantly higher for patients who stopped at 6 cycles than those who continued the treatment (Table 4). Unfortunately, among the patients who continued the treatment after 6 cycles, we can not identify which subgroup of patients benefited most from continuation of treatment despite methodological efforts. One of the main hypothesis could be low activity of MGMT via methylation of its promoter. And, we can hypothesize that persistence of an enhanced lesion on MRI after 6 cycles, and/or positive methylated status, might be one of the best indication.

\section{Perspective}

Few countries have national registry for brain tumors (e.g., USA, Scandinavian countries, Austria, etc.). Sometimes they participate in international works about epidemiology, biology, or genetic analysis, but to our knowledge international population-based study analyzing oncological management for the entire population of several countries does not exist. International population-based studies analyzing oncological management for brain tumors (e.g., GBM) would be an opportunity to answer clinical questions.

\section{Conclusions}

To our knowledge, this is the first work detailing the firstline treatment including the duration of maintenance therapy of temozolomide, and survival in a large GBM patient population-based study. We showed that prolonging the adjuvant temozolomide beyond 6 cycles in non-progressive patient positively impacted survival. Complementary analysis including adverse effects of the treatments, quality of life, and biology, warrant a dedicated randomized clinical trial, or a large prospective international population-based study.

Acknowledgements We thank patients and families, and all pathologists, neurosurgeons, neurologists, oncologists, general practitioners, biostatisticians, clinical research technicians, students, and all those who participated in this important collaborative work. We are grateful to Sarah Kabani for editorial assistance (salaried position funded by the CHU-Nimes, in accordance with Good Publication Practice-GPP3 guidelines).

Funding This work was conducted with the financial support of Grants from the French Institut National du Cancer-INCa-, Ligue Nationale Contre le Cancer, Associations pour la Recherche sur les Tumeurs Cérébrales, and Département de l'Hérault.

\section{Compliance with ethical standards}

Conflict of interest None of the authors have any competing interests.

OpenAccess This article is distributed under the terms of the Creative Commons Attribution 4.0 International License (http://creativeco mmons.org/licenses/by/4.0/), which permits unrestricted use, distribution, and reproduction in any medium, provided you give appropriate credit to the original author(s) and the source, provide a link to the Creative Commons license, and indicate if changes were made.

\section{References}

1. Ostrom QT, Bauchet L, Davis FG et al (2014) The epidemiology of glioma in adults: a "state of the science" review. Neurooncology 16(7):896-913

2. Ostrom QT, Gittleman H, Liao P et al (2017) CBTRUS Statistical Report: primary brain and other central nervous system tumors diagnosed in the United States in 2010-2014. Neuro-oncology 19(S_5):v1-v88

3. Bauchet L, Zouaoui S, Darlix A et al (2014) Assessment and treatment relevance in elderly glioblastoma patients. Neuro-oncology 16(11):1459-1468

4. Laperriere N, Zuraw L, Cairncross G, Cancer Care Ontario Practice Guidelines Initiative Neuro-Oncology Disease Site Group (2002) Radiotherapy for newly diagnosed malignant glioma in adults: a systematic review. Radiother Oncol 64(3):259-273

5. Stewart LA (2002) Chemotherapy in adult high-grade glioma: a systematic review and meta-analysis of individual patient data from 12 randomised trials. Lancet 359(9311):1011-1018

6. Stupp R, Mason WP, van den Bent MJ et al (2005) Radiotherapy plus concomitant and adjuvant temozolomide for glioblastoma. $\mathrm{N}$ Engl J Med 352(10):987-996

7. Stummer W, Pichlmeier U, Meinel T et al (2006) Fluorescenceguided surgery with 5-aminolevulinic acid for resection of malignant glioma: a randomised controlled multicentre phase III trial. Lancet Oncol 7(5):392-401

8. Chaichana KL, Jusue-Torres I, Navarro-Ramirez R et al (2014) Establishing percent resection and residual volume thresholds affecting survival and recurrence for patients with newly diagnosed intracranial glioblastoma. Neuro-oncology 16(1):113-122

9. Brown TJ, Brennan MC, Li M et al (2016) Association of the extent of resection with survival in glioblastoma: a systematic review and meta-analysis. JAMA Oncol 2(11):1460-1469

10. Stupp R, Taillibert S, Kanner A et al (2017) Effect of tumortreating fields plus maintenance temozolomide vs maintenance temozolomide alone on survival in patients with glioblastoma: a randomized clinical trial. JAMA 318(23):2306-2316

11. Westphal M, Hilt DC, Bortey E et al (2003) A phase 3 trial of local chemotherapy with biodegradable carmustine (BCNU) wafers (Gliadel wafers) in patients with primary malignant glioma. Neuro-oncology 5(2):79-88

12. Pallud J, Audureau E, Noel G et al (2015) Long-term results of carmustine wafer implantation for newly diagnosed glioblastomas: a controlled propensity-matched analysis of a French multicenter cohort. Neuro-oncology 17(12):1609-1619

13. NCCN (2017) Clinical practice guidelines in oncology: central nervous system cancers version 1.2017. https://www.nccn.org/ professionals/physician_gls/pdf/cns.pdf. Accessed 29 Jan 2018

14. Sage W, Guilfoyle M, Luney C et al (2018) Local alkylating chemotherapy applied immediately after 5-ALA guided resection of glioblastoma does not provide additional benefit. J Neurooncol 136(2):273-280 
15. Chinot OL, Wick W, Mason W et al (2014) Bevacizumab plus radiotherapy-temozolomide for newly diagnosed glioblastoma. N Engl J Med 370(8):709-722

16. Gilbert MR, Dignam JJ, Armstrong TS et al (2014) A randomized trial of bevacizumab for newly diagnosed glioblastoma. N Engl J Med 370(8):699-708

17. Woehrer A, Bauchet L, Barnholtz-Sloan JS (2014) Glioblastoma survival: has it improved? Evidence from population-based studies. Curr Opin Neurol 27(6):666-674

18. Bauchet L, Rigau V, Mathieu-Daudé $\mathrm{H}$ et al (2007) French brain tumor data bank: methodology and first results on 10,000 cases. J Neurooncol 84(2):189-199

19. Bauchet L, Mathieu-Daudé H, Fabbro-Peray P et al (2010) Oncological patterns of care and outcome for 952 patients with newly diagnosed glioblastoma in 2004. Neuro-oncology 12(7):725-735

20. Rigau V, Zouaoui S, Mathieu-Daudé $\mathrm{H}$ et al (2011) French brain tumor database: 5-year histological results on 25756 cases. Brain Pathol 21(6):633-644

21. Scott JG, Bauchet L, Fraum TJ et al (2012) Recursive partitioning analysis of prognostic factors for glioblastoma patients aged 70 years or older. Cancer 118(22):5595-5600

22. Zouaoui S, Darlix A, Fabbro-Peray P et al (2014) Oncological patterns of care and outcomes for 265 elderly patients with newly diagnosed glioblastoma in France. Neurosurg Rev 37(3):415-423; discussion 423-424

23. Darlix A, Zouaoui S, Virion J-M et al (2014) Significant heterogeneity in the geographical distribution of diffuse grade II/III gliomas in France. J Neurooncol 120(3):547-555

24. Zouaoui S, Darlix A, Rigau V et al (2018) Descriptive epidemiology of 13,038 newly diagnosed and histologically confirmed meningiomas in France: 2006-2010. Neurochirurgie 64(1):15-21

25. Terrier L-M, Bauchet L, Rigau V et al (2017) Natural course and prognosis of anaplastic gangliogliomas: a multicenter retrospective study of 43 cases from the French Brain Tumor Database. Neuro-oncology 19(5):678-688

26. Darlix A, Zouaoui S, Rigau V et al (2017) Epidemiology for primary brain tumors: a nationwide population-based study. J Neurooncol 131(3):525-546

27. Louis DN, Ohgaki H, Wiestler OD, Cavenee WK (2007) WHO classification of tumours of the central nervous system, vol 1 , 4th edn. IARC Press, Lyon

28. Gramatzki D, Dehler S, Rushing EJ et al (2016) Glioblastoma in the Canton of Zurich, Switzerland revisited: 2005 to 2009. Cancer 122(14):2206-2215

29. Zhu P, Du XL, Lu G, Zhu J-J (2017) Survival benefit of glioblastoma patients after FDA approval of temozolomide concomitant with radiation and bevacizumab: a population-based study. OncoTarget 8(27):44015-44031
30. Hansen S, Rasmussen BK, Laursen RJ et al (2018) Treatment and survival of glioblastoma patients in Denmark: the Danish NeuroOncology Registry 2009-2014. J Neurooncol 139(2):479-489

31. Stupp R, Hegi ME, Mason WP et al (2009) Effects of radiotherapy with concomitant and adjuvant temozolomide versus radiotherapy alone on survival in glioblastoma in a randomised phase III study: 5-year analysis of the EORTC-NCIC trial. Lancet Oncol 10(5):459-466

32. Graus F, Bruna J, Pardo J et al (2013) Patterns of care and outcome for patients with glioblastoma diagnosed during 2008-2010 in Spain. Neuro-oncology 15(6):797-805

33. Brandes AA, Franceschi E, Ermani M et al (2014) Pattern of care and effectiveness of treatment for glioblastoma patients in the real world: results from a prospective population-based registry. Could survival differ in a high-volume center? Neurooncol Pract 1(4):166-171

34. van den Bent MJ, Baumert B, Erridge SC et al (2017) Interim results from the CATNON trial (EORTC study 26053-22054) of treatment with concurrent and adjuvant temozolomide for $1 \mathrm{p} / 19 \mathrm{q}$ non-co-deleted anaplastic glioma: a phase 3, randomised, openlabel intergroup study. Lancet 390(10103):1645-1653

35. Blumenthal DT, Gorlia T, Gilbert MR et al (2017) Is more better? The impact of extended adjuvant temozolomide in newly diagnosed glioblastoma: a secondary analysis of EORTC and NRG Oncology/RTOG. Neuro-oncology 19(8):1119-1126

36. Perry JR, Laperriere N, O'Callaghan CJ et al (2017) Short-course radiation plus temozolomide in elderly patients with glioblastoma. N Engl J Med 376(11):1027-1037

37. Darlix A, Baumann C, Lorgis V et al (2013) Prolonged administration of adjuvant temozolomide improves survival in adult patients with glioblastoma. Anticancer Res 33(8):3467-3474

38. Roldán Urgoiti GB, Singh AD, Easaw JC (2012) Extended adjuvant temozolomide for treatment of newly diagnosed glioblastoma multiforme. J Neurooncol 108(1):173-177

39. Seiz M, Krafft U, Freyschlag CF et al (2010) Long-term adjuvant administration of temozolomide in patients with glioblastoma multiforme: experience of a single institution. J Cancer Res Clin Oncol 136(11):1691-1695

40. Barbagallo GMV, Paratore S, Caltabiano R et al (2014) Longterm therapy with temozolomide is a feasible option for newly diagnosed glioblastoma: a single-institution experience with as many as 101 temozolomide cycles. Neurosurg Focus 37(6):E4

41. Skardelly M, Dangel E, Gohde J et al (201) Prolonged temozolomide maintenance therapy in newly diagnosed glioblastoma. Oncologist 22(5):570-575

42. Gramatzki D, Kickingereder P, Hentschel B et al (2017) Limited role for extended maintenance temozolomide for newly diagnosed glioblastoma. Neurology 88(15):1422-1430

\section{Affiliations}

\section{Pascale Fabbro-Peray ${ }^{1,2}$. Sonia Zouaoui ${ }^{3,4,5}$. Amélie Darlix ${ }^{5,6} \cdot$ Michel Fabbro $^{5,6}$. Johan Pallud ${ }^{7}$. Valérie Rigau ${ }^{5,8}$. Hélène Mathieu-Daude ${ }^{5,9} \cdot$ Faiza Bessaoud $^{10}$. Fabienne Bauchet ${ }^{5}$. Adeline Riondel $^{1,2}$. Elodie Sorbets ${ }^{1,2}$. Marie Charissoux $x^{5,11}$. Aymeric Amelot ${ }^{12} \cdot$ Emmanuel Mandonnet $^{12} \cdot$ Dominique Figarella-Branger $^{13}$. Hugues Duffau ${ }^{3,4,5} \cdot$ Brigitte Tretarre $^{10} \cdot$ Luc Taillandier $^{14} \cdot$ Luc Bauchet $^{3,4,5}$ (i)}

Luc Bauchet

1-bauchet@chu-montpellier.fr

1 Department of Biostatistics, Epidemiology, Public Health, CHU Nîmes, Nîmes, France
2 EA2415 Research Unit, Montpellier University, Montpellier, France

3 Department of Neurosurgery, Hopital Gui de Chauliac, CHU Montpellier, Montpellier University Medical Center, 80 Avenue Fliche, 34295 Montpellier, France 
4 INSERM U1051, Montpellier, France

5 Groupe de Neuro-Oncologie du Languedoc Roussillon, ICM, Montpellier, France

6 Department of Medical Oncology, ICM, Montpellier, France

7 Department of Neurosurgery, Sainte Anne Hospital, and University Paris Descartes, Paris, France

8 Department of Neuropathology, Hopital Gui de Chauliac, CHU Montpellier, Montpellier University Medical Center, Montpellier, France

9 Department of Medical informatics, ICM, Montpellier, France
10 Registre des Tumeurs de l'Hérault, ICM, Montpellier, France

11 Department of Radiation Oncology, ICM, Montpellier, France

12 Department of Neurosurgery, CHU Lariboisière, Paris, France

13 Department of Neuropathology, CHU Marseille, INSERM U911, Marseille, France

14 Department of Neuro-oncology, CHU Nancy, Nancy, France 